Pacific Journal of Mathematics

ON THE RETRACTABILITY OF SOME ONE-RELATON 


\title{
ON THE RETRACTABILITY OF SOME ONE-RELATOR GROUPS
}

\author{
Richard D. BYRD, Justin T. LLOYD \\ AND Roberto A. MENA
}

\begin{abstract}
Recently the concept of a retractable group has been introduced. This class of groups contains the class of latticeordered groups as a proper subclass, and, in particular, contains the class of all torsion-free abelian groups. Retractable groups enjoy many of the properties of lattice-ordered groups; in fact, most results concerning lattice-ordered groups have immediate extensions to this wider class. In this note, we investigate the retractability of certain two-generator one-relator groups.
\end{abstract}

1. Introduction. There has been an abundance of literature on the class of groups presented by a single defining relation and, in particular, on the groups given by the presentation

$$
\left\langle a, c \mid a^{-1} c^{m} a=c^{n}\right\rangle,
$$

where $m$ and $n$ are integers. In [1] the concept of a retractable group was introduced and in this note we attempt to determine which of this latter class of groups are retractable.

In Theorem 3.3 we show that the groups $\left\langle a, c \mid a^{-1} c a=c^{m}\right\rangle$, where $m$ is a positive integer, are retractable and each admits at least a countably infinite number of retractions that satisfy condition

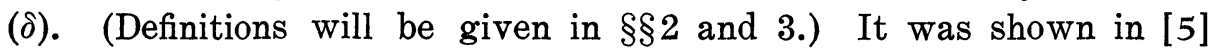
that the group $\left\langle a, c \mid a^{-1} c a=c^{2}\right\rangle$ admits exactly four full orders. Each of these induces a retraction on this group. We show in Theorem 3.5 that each of the groups $\left\langle a, c \mid a^{-1} c a=c^{m}\right\rangle$, where $m>1$, admits exactly four lattice-orders and each of these is a full order. In Theorem 3.6 we show that the groups $\left\langle a, c \mid a^{-1} c a=c^{m}\right\rangle$, where $m<0$, admit retractions if and only if 2 is a factor of $m$, and in this case, none of these groups admit lattice-orders. In Theorem 3.1 we show that if $G$ is a retractable group and $g^{n}=h^{n}$, for some $g, h \in G$ and some natural number $n$, then $g$ and $h$ are conjugate. As a corollary to this theorem, we are able to show that the groups $\left\langle a, c \mid a^{n}=c^{n}\right\rangle$, where $n$ is a natural number and $n>1$, and

$$
\left\langle a, c \mid a^{-1} c^{m} a=c^{n}\right\rangle,
$$

where $m$ and $n$ are distinct integers and $\operatorname{gcd}(m, n)>1$, are not retractable. 
2. Preliminaries. In this section we shall recall some definitions and establish some notation that we shall use for the remainder of this note. For a group $G$, let $F(G)$ denote the collection of all finite, nonempty subsets of $G$. Then $F(G)$ is a join monoid, where the join operation is set union and the binary operation is multiplication of complexes. A homomorphism $\sigma$ of the monoid $F(G)$ into $G$ such that $\{g\} \sigma=g$ for every $g$ in $G$ will be called a retraction of $G$. We denote by Ret $G$ the set of all retractions of $G$. If Ret $G$ is nonempty, then $G$ is said to be a retractable group. It follows immediately from the definition of a retraction that if $\sigma \in \operatorname{Ret} G, A \in F(G)$, and $x, y \in G$, then $x(A \sigma) y=(x A y) \sigma$. If $\sigma \in \operatorname{Ret} G$ and $H$ is a subgroup of $G$ such that $\sigma \mid F(H) \in \operatorname{Ret} H$, then $H$ is said to be a $\sigma$ subgroup.

Let $G$ be a lattice-ordered group and define $\sigma$ from $F(G)$ into $G$ by $A \sigma=\bigvee A$ for every $A \in F(G)$. Then $\sigma \in \operatorname{Ret} G$ [1, Theorem 2.1] and $\sigma$ is called the retraction of $G$ induced by the lattice-ordering of $G$. Thus, the class of lattice-ordered groups is a subclass of the class of retractable groups and, as indicated in the introduction, it is a proper subclass. If $\sigma \in \operatorname{Ret} G$ and $\{A \mid A \in F(G)$ and $A \sigma=i\}$, where $i$ denotes the identity of $G$, is a convex subsemilattice of $F(G)$, then there is a lattice-ordering of $G$ such that $A \sigma=\mathrm{V} A$ for every $A \in F(G)$ [1, Theorem 3.2]. The class of retractable groups is a proper subclass of the class of torsion free groups [1, Theorem 2.2 and Example 2.7].

If $\sigma \in \operatorname{Ret} G$ and $H$ is a subgroup of $G$, then $H$ is said to be a $\rho$ - $\sigma$-subgroup (resp., $\lambda$ - $\sigma$-subgroup) if $A=\left\{a_{1}, \cdots, a_{n}\right\} \in F(G)$ and $h_{1}, \cdots, h_{n} \in H$ implies that $H(A \sigma)=H\left(\left\{h_{1} a_{1}, \cdots, h_{n} a_{n}\right\} \sigma\right)$ (resp., $(A \sigma) H=$ $\left.\left(\left\{a_{1} h_{1}, \cdots, a_{n} h_{n}\right\} \sigma\right) H\right)$. In [1] and [2] a $\rho$ - $\sigma$-subgroup was called a $c$ $\sigma$-subgroup. A subgroup which is both a $\rho$ - $\sigma$-subgroup and a $\lambda-\sigma$ subgroup will be called a solid $\sigma$-subgroup. Clearly a normal $\rho-\sigma$ subgroup is a solid $\sigma$-subgroup. It was shown in [1, Theorem 4.2] that the collection of all $\rho$ - $\sigma$-subgroups of $G$ is a complete sublattice of the lattice of all subgroups of $G$. If $H$ is a $\rho$ - $\sigma$-subgroup, then $H$ satisfies the purity condition in the sense that $g^{n} \in H$, for some $g \in G$ and some natural number $n$, implies $g \in H$ [1, Corollary 4.10]. If $G$ is a lattice-ordered group, $\sigma$ is the retraction of $G$ induced by the lattice-ordering of $G$, and $H$ is a subgroup of $G$, then $H$ is a $\rho$ - $\sigma$-subgroup if and only if $H$ is a convex $l$-subgroup of $G[1$, Theorem 4.7].

We shall denote the natural numbers, integers, and rational numbers by $N, Z$, and $Q$ respectively. If $X$ and $Y$ are sets, $X \backslash Y$ will denote the set of elements in $X$ but not in $Y$.

If $m \in Z \backslash\{0\}$, let $Q_{m}=\left\{n m^{-k} \mid n, k \in Z\right\}$ and $G_{m}=Q_{m} \times Z$. For $(a, x),(b, y) \in G_{m}$, define $(a, x)+(b, y)=\left(m^{y} a+b, x+y\right)$. Then $G_{m}$ is 
a group and is a splitting extension of $Q_{m}$ by $Z$. If $H_{m}=Q_{m} \times\{0\}$, then $H_{m}$ is a normal subgroup of $G_{m}$. If $G=\left\langle a, c \mid a^{-1} c a=c^{m}\right\rangle$, then each $g$ in $G$ can be expressed as $g=a^{r} c^{s} a^{t}$, where $r, s, t \in Z$ and $t<0$. It is easily established that the mapping that sends $a^{r} c^{s} a^{t}$ onto $(0, r)+(s, 0)+(0, t)=\left(m^{t} s, r+t\right)$ is an isomorphism of $G$ onto $G_{m}$. In [2], Ret $Q_{m}$ was classified and for this reason, we shall identity $G$ with $G_{m}$ in $\S 3$.

3. One relator groups. We begin this section by showing that the class of retractable groups is a subclass of the class of power conjugate groups.

THEOREM 3.1. If $G$ is a retractable group and $g, h \in G$ such that $g^{n}=h^{n}$ for some $n \in N$, then $g$ and $h$ are conjugate.

Proof. Let $\sigma \in \operatorname{Ret} G$ and $\left\{g^{n-1}, g^{n-2} h, \cdots, g h^{n-2}, h^{n-1}\right\} \sigma=a$. Then

$$
\begin{aligned}
g a & =g\left(\left\{g^{n-1}, g^{n-2} h, \cdots, g h^{n-2}, h^{n-1}\right\} \sigma\right)=\left\{g^{n}, g^{n-1} h, \cdots, g^{2} h^{n-2}, g h^{n-1}\right\} \sigma \\
& =\left\{h^{n}, g^{n-1} h, \cdots, g^{2} h^{n-2}, g h^{n-1}\right\} \sigma=\left(\left\{h^{n-1}, g^{n-1}, \cdots, g^{2} h^{n-3}, g h^{n-2}\right\} \sigma\right) h \\
& =a h .
\end{aligned}
$$

Therefore, $g=a h a^{-1}$.

CoROllary 3.2. (i) Let $m, n \in Z$ with $m \neq n$ and $\operatorname{gcd}(m, n)=$ $d>1$. If $G=\left\langle a, c \mid a^{-1} c^{m} a=c^{n}\right\rangle$, then $G$ is not retractable.

(ii) If $n \in N$ with $n>1$ and $G=\left\langle a, c \mid a^{n}=c^{n}\right\rangle$, then $G$ is not retractable.

Proof. (i ) Let $F$ be the free group on the generators $a$ and $c$, and suppose that $n>m$. Let $t=n-m, Z_{t}$ denote the group of integers modulo $t$, and $\phi$ be the homomorphism of $F$ into $Z_{t}$ given by $a \phi=0$ and $c \phi=1$. Then $\left(a^{-1} c^{m} a\right) \dot{\phi}=m$ and $\left(c^{n}\right) \phi=n$ and since $m-n=0$ in $Z_{t}, a^{-1} c^{m} a c^{-n} \in \operatorname{Ker} \phi$. It follows that $Z_{t}$ is a homomorphic image of $G$. Let $r, s \in Z$ so that $r d=m$ and $s d=n$. Then $\left(a^{-1} c^{r} a\right)^{d}=\left(c^{s}\right)^{d}, \operatorname{gcd}(r, s)=1$, and since $m \neq n, r \neq s$. Consequently, $r$ and $s$ are distinct elements in $Z_{t}$ and hence, can not be conjugate in $Z_{t}$. Therefore, $a^{-1} c^{r} a$ and $c^{s}$ are not conjugate in $G$. By the theorem, $G$ is not retractable.

(ii) It is readily verified that $a$ and $c$ are not conjugate in $G$ and hence, $G$ is not retractable.

A retraction $\sigma$ of $G$ is said to satisfy $(\delta)$ provided that $\left\{g_{1}, \cdots, g_{m}\right\} \in F(G)$ and $n \in N$ implies $\left\{g_{1}^{n}, \cdots, g_{m}^{n}\right\} \sigma=\left(\left\{g_{1}, \cdots, g_{m}\right\} \sigma\right)^{n}$. If $G$ is abelian and $\sigma \in \operatorname{Ret} G$, then $\sigma$ satisfies $(\delta)$ [2, Corollary 3.4]. If $G$ is a lattice-ordered group and $\sigma$ is the retraction induced by the lattice-ordering of $G$, then $\sigma$ satisfies $(\delta)$ if and only if the 
lattice-ordering is representable (see [4, Theorem 1.8]).

Let $H$ be a subgroup of $Q$ and $H^{\perp}=\{r \mid r \in Q$ and $r h \in H$ for every $h \in H\}$. It was shown in [2, Corollary 3.12] that if $H$ is a nonzero subgroup of $Q$, then $\operatorname{Ret} H=\left\{\sigma_{r} \mid r \in H^{\perp}\right\}$, where

$$
A \sigma_{r}=(r+1) \max A-r \min A
$$

for all $A \in F(H)$. Since $Z \subseteq H^{\perp}$, Ret $H$ is countably infinite and hence so is $\operatorname{Ret} H_{m}, m \in N$. A corollary of the next result is that Ret $G_{m}$ is at least countably infinite.

TheOREM 3.3. If $\tau \in \operatorname{Ret} H_{m}$, where $m \in N$, then $\tau$ has an extension to a retraction $\sigma$ of $G_{m}$ that satisfies $(\delta)$.

Proof. If $m=1$, then $G_{m}$ is abelian, $H_{m}$ is a direct summand of $G_{m}$, and the verification of the theorem is routine. Thus, we assume that $m>1$. Let $\tau \in \operatorname{Ret} H_{m}$ and $A=\left\{\left(a_{1}, 0\right), \cdots,\left(a_{t}, 0\right)\right\} \in$ $F\left(H_{m}\right)$, where $a_{1}<\cdots<a_{t}$. Then, by the above remarks, $A \tau=$ $\left((r+1) a_{t}-r a_{1}, 0\right)$ for some $r \in Q_{m}^{\perp}$. Next we show that if $g \in G_{m}$, $-g+A \tau+g=(-g+A+g) \tau$ and to prove this, it suffices to take $g=(0, x)$, where $x \in Z$. Now

$$
-(0, x)+A \tau+(0, x)=\left(m^{x}\left((r+1) a_{t}-r a_{1}\right), 0\right) .
$$

Since $m^{x}>0, m^{x} a_{1}<\cdots<m^{x} a_{t}$ and hence, $(-(0, x)+A+(0, x)) \tau=$ $\left((r+1) m^{x} a_{t}-r m^{x} a_{1}, 0\right)$. Since $G_{m} / H_{m}$ can be fully ordered, we have that $\tau$ has an extension to a retraction $\sigma$ of $G_{m}$ and that $H_{m}$ is a $\rho$ - $\sigma$-subgroup of $G_{m}$ [2, Theorem 3.18]. We explicitly describe the construction of $\sigma$ below, so that it may be proven that $\sigma$ satisfies $(\delta)$.

Let $A=\left\{\left(a_{1}, x_{1}\right), \cdots,\left(a_{t}, x_{t}\right)\right\} \in F\left(G_{m}\right), \quad$ where $\quad x_{1} \leqq \cdots \leqq x_{p-1}<$ $x_{p}=\cdots=x_{t}$ and $a_{p}<\cdots<a_{t}, B=\left\{\left(a_{p}, x_{p}\right), \cdots,\left(a_{t}, x_{t}\right)\right\}-\left(a_{t}, x_{t}\right)$, $s \in N$, and $C=\left\{s\left(a_{1}, x_{1}\right), \cdots, s\left(a_{t}, x_{t}\right)\right\}$. It was shown in the proof of [2, Theorem 3.18] that $A \sigma=B \tau+\left(a_{t}, x_{t}\right)=\left((r+1) a_{t}-r a_{p}, x_{t}\right)$. Consequently, $s(A \sigma)=\left(\sum_{j=1}^{s} m^{(j-1) x} t\left((r+1) a_{t}-r a_{p}\right), s x_{t}\right)$. On the other hand, $C \sigma=\left((r+1)\left(\sum_{j=1}^{s} m^{(j-1) x} a_{t}\right)-r\left(\sum_{j=1}^{s} m^{(j-1) x} a_{p}\right), s x_{t}\right)$ and it follows that $\sigma$ satisfies $(\delta)$.

If $\sigma \in \operatorname{Ret} G, H$ is a normal $\rho$ - $\sigma$-subgroup of $G, X=\left\{H g_{1}, \cdots, H g_{t}\right\} \in$ $F(G / H)$, and $X \sigma^{*}=H\left(\left\{g_{1}, \cdots, g_{t}\right\} \sigma\right)$, then $\sigma^{*} \in \operatorname{Ret} G / H$ [1, Theorem 4.3]. The following example shows that if $\sigma \in \operatorname{Ret} G, H$ is a normal $\rho$ - $\sigma$-subgroup of $G, \sigma \mid F(H)$ and $\sigma^{*}$ satisfy $(\delta)$, then $\sigma$ need not satisfy $(\delta)$.

ExAmple 3.4. Let $K=Z \times Z \times Z$ and define

$$
(a, b, c)+(x, y, z)= \begin{cases}(a+x, b+y, c+z) & \text { if } z \text { is even } \\ (b+x, a+y, c+z) & \text { if } z \text { is odd } .\end{cases}
$$


Then $K$ is a group and a splitting extension of $Z \times Z$ by $Z$. Define $(a, b, c) \geqq(0,0,0)$ if $c>0$, or if $c=0, a \geqq 0$, and $b \geqq 0$. Then $K$ is a nonrepresentable lattice-ordered group and hence, if $\sigma$ is the retraction induced by the lattice-ordering of $K$, then $\sigma$ does not satisfy $(\delta)$. If $J=Z \times Z \times\{0\}$, then $J$ is an abelian normal $\rho$ - $\sigma$-subgroup of $K$ and $K / J$ is abelian. Thus, $\sigma \mid F(J)$ and $\sigma^{*}$ satisfy $(\delta)$.

We note in passing that it is not difficult to give an example of a group $G$, distinct retractions $\sigma$ and $\tau$ of $G$, and a normal subgroup $H$ of $G$ such that $H$ is both a $\rho$ - $\sigma$-subgroup and a $\rho$ - $\tau$-subgroup, $\sigma|F(H)=\tau| F(H)$, and $\sigma^{*}=\tau^{*}$.

In [5, Theorem 2] Fuchs and Sasiada showed that $G_{2}$ is an $0^{*}$ group with exactly four different full orders and that $H_{2}$ is a convex subgroup in each of these orders. If $m \in N$ with $m>1$, then $H_{m}$ is an abelian normal subgroup of $G_{m}$ such that $G_{m} / H_{m}$ is abelian and such that for each $a \in H_{m}$ and $b \in G_{m} \backslash H_{m}$, there exist distinct positive integers $s$ and $t$ so that $-b+s a+b=t a$. Kargapolov [6, p. 17] proved that a group that satisfied these conditions had the property that each full order for any subgroup can be extended to a full order of the group. A group satisfying this property is an $0^{*}$-group (see [7] or [9]). Although Theorem 3.3 (and the discussion preceding it) showed that $G_{m}$ has at least a countably infinite number of retractions, our next theorem shows that $G_{m}$ has exactly four latticeorders and each of these is a full ordering.

THEOREM 3.5. Let $m \in Z$ and $\sigma \in \operatorname{Ret} G_{m}$.

(i) If $m \neq 1$ and $H$ is a proper solid $\sigma$-subgroup of $G_{m}$, then $H=H_{m}$.

(ii) If $m>1$, then each lattice-ordering of $G_{m}$ is a full ordering and $G_{m}$ has exactly four full orders.

Proof. (i) First we show that $G_{m}$ can have at most one proper solid $\sigma$-subgroup. Let $H$ and $J$ be distinct nonzero solid $\sigma$ subgroups of $G_{m}$.

Case 1. $J$ is properly contained in $H$. If $(a, 0) \in J$ for some $a \neq 0$, then by the purity of $J,(1,0) \in J$. Since $J$ is properly contained in $H$ and $H$ is pure, it follows that $(0,1) \in H$. Therefore, $G_{m}=H$. Consequently, we may assume that for each $(a, b) \in$ $J \backslash\{(0,0)\}, b \neq 0$. If $(c, 0) \in H$ for some $c \neq 0$, then by the preceding argument, we again have $G_{m}=H$. Thus, we may further assume that for each $(c, d) \in H \backslash J, d \neq 0$. Let $(a, b) \in J \backslash\{(0,0)\}$ and $(c, d) \in H \backslash J$. By the purity of $J, b(c, d) \neq d(a, b)$. But then $(0 ; 0) \neq(h, 0)=$ $b(c, d)-d(a, b) \in H$, a contradiction. 
Case 2. $J$ is not properly contained in $H$. If $H \cap J \neq\{(0,0)\}$, then $H \cap J$ is a nonzero solid $\sigma$-subgroup properly contained in $J$. By Case $1, J=G_{m}$. Suppose (by way of contradiction) that $H \cap J=$ $\{(0,0)\}$. Then the subgroup $K$ generated by $H$ and $J$ is their direct sum [3, Corollary 4.3]. Since $G_{m}$ is subdirectly irreducible, $K$ is a proper solid $\sigma$-subgroup of $G_{m}$ and $K$ properly contains $J$. But by Case $1, K=G_{m}$, a contradiction.

Therefore, $G_{m}$ can have at most one proper solid $\sigma$-subgroup and necessarily, this subgroup must be normal in $G_{m}$. The only proper subgroup with these properties is $H_{m}$. We note for $m \neq 0,1$ that $H_{m}$ is the centralizer of $(1,0)$ in $G_{m}$. Hence, by [1, Theorem 2.14], $H_{m}$ is always a $\sigma$-subgroup of $G_{m}$.

(ii) Let $\sigma$ be the retraction induced by a lattice-ordering of $G_{m}$. Then each convex $l$-subgroup is a solid $\sigma$-subgroup. By (i) the convex $l$-subgroups that contain $\{(0,0)\}$ form a chain. Consequently, $G_{m}$ is a fully ordered group [4, Theorem 1.7].

As in [5], $G_{m}$ has at least the four full orders corresponding to the cases $(0,0)<(1,0)<(0,1), \quad(0,0)<(-1,0)<(0,1), \quad(0,0)<$ $(1,0)<(0,-1)$, and $(0,0)<(-1,0)<(0,-1)$. Since $m>1, G_{m}$ is nonabelian. Hence, by Hölder's theorem (see [4, p. 0.24]), $G_{m}$ must have a proper convex subgroup in any full ordering. By (i), this subgroup must be $H_{m}$. It follows that $G_{m}$ can have no more than the four full orders given above.

We shall show below that if $m$ is negative, then $G_{m}$ admits no lattice-orders. It was noted in [5] that $G_{1}$ has continuously many full orders. Since $G_{1}$ is the direct product of two copies of $Z$, it also admits retractions $\sigma$ for which there are two proper $\rho-\sigma$-subgroups.

It was shown in [1, Theorem 5.1] that if $\phi$ is an automorphism or an anti-automorphism of $G$ and $\sigma \in \operatorname{Ret} G$, then $\phi \sigma \phi^{-1} \in \operatorname{Ret} G$. If $\phi$ is the anti-automorphism of $G$ given by $g \phi=g^{-1}$, then the retraction $\sigma^{\prime}=\dot{\phi} \sigma \dot{\phi}^{-1}$ is called the dual of $\sigma$. If $G$ is a lattice-ordered group and $\sigma$ is the retraction induced by the lattice-ordering of $G$, then $\sigma^{\prime}$ induces a lattice-ordering of $G$ which is the dual of the given lattice-ordering [1, Corollary 5.2]. If $\sigma=\sigma^{\prime}$, then we say that $\sigma$ is self dual. If $H$ is a two-divisible subgroup of $Q$, then, as noted earlier, $\sigma_{-1 / 2} \in \operatorname{Ret} H$ and it is easily verified that $\sigma_{-1 / 2}$ is self dual. In fact, $\sigma_{-1 / 2}$ is the only self dual retraction of $H$. (Any two-divisible torsion free abelian group admits a self dual retraction.)

THEOREM 3.6. Let $m$ be a negative integer. Then $G_{m}$ is retractable if and only if 2 divides $m$. Moreover,

(i ) if $\sigma \in \operatorname{Ret} G_{m}$, then $H_{m}$ is a $\sigma$-subgroup of $G_{m}$ and $\sigma \mid F\left(H_{m}\right)$ is self dual; 
(ii) $G_{m}$ is not lattice-orderable;

(iii) if 2 divides $m$ and $\tau$ is the self dual retraction of $H_{m}$, then $\tau$ has an extension to a retraction $\sigma$ of $G_{m}$ that satisfies $(\delta)$ and $H_{m}$ is a proper $\rho$ - $\sigma$-subgroup of $G_{m}$.

Proof. Suppose that $G_{m}$ is retractable and let $\sigma \in \operatorname{Ret} G_{m}$. We observed in the proof of (i) of Theorem 3.5 that $H_{m}$ is a $\sigma$-subgroup of $G$. Hence, $\{(0,0),(1,0)\} \sigma=\left(n m^{-k}, 0\right)$ for some $n, k \in Z$ with $k \geqq 0$. Thus,

$$
\begin{aligned}
\left(n m^{-k+1}, 0\right) & =-(0,1)+\left(n m^{-k}, 0\right)+(0,1) \\
& =\{(0,0),-(0,1)+(1,0)+(0,1)\} \sigma \\
& =\{(0,0),(m, 0)\} \sigma .
\end{aligned}
$$

By [1, Theorem 2.4],

$$
\{(0,0),(m, 0)\} \sigma=(-m)\left(n m^{-k}, 0\right)+(m, 0)=\left(m-n m^{-k+1}, 0\right) .
$$

Therefore, $2 n=m^{k}$ and it follows that 2 divides $m$. Also, we have $(1 / 2,0)=\{(0,0),(1,0)\} \sigma$. It was shown in [1, Example 5.7] that the image of $\{(0,0),(1,0)\}$ under $\sigma$ completely determines $\sigma \mid F\left(H_{m}\right)$. Consequently, $\sigma \mid F\left(H_{m}\right)$ is self dual and we have proven (i).

If $G_{m}$ is lattice-orderable and $\sigma$ is the retraction of $G_{m}$ induced by the lattice-ordering, then $(1 / 2,0)=\{(0,0),(1,0)\} \sigma$ must be positive. Since $m<0,(-m / 2,0)$ would also be positive. But $(m / 2,0)=$ $-(0,1)+(1 / 2,0)+(0,1)$ and hence, the positive cone of $G$ contains a nonzero element and its inverse, which is impossible. Therefore, we have proven (ii).

Next suppose that 2 divides $m$. For $A=\left\{\left(a_{1}, 0\right), \cdots,\left(a_{t}, 0\right)\right\} \in$ $F\left(H_{m}\right)$, define $A \tau=\left(\left\{a_{1}, \cdots, a_{t}\right\} \sigma_{-1 / 2}, 0\right)$. Then $\tau$ is a self dual retraction of $H_{m}$. To show that for every $g \in G_{m}$,

$$
-g+(A \tau)+g=(-g+A+g) \tau,
$$

it suffices to take $g=(0, x)$, where $x \in Z$. Now $(0,-x)+A \tau+(0, x)=$ $\left(m^{x}\left(\left\{a_{1}, \cdots, a_{t}\right\} \sigma_{-1 / 2}\right), 0\right)=\left(\left\{m^{x} a_{1}, \cdots, m^{x} a_{t}\right\} \sigma_{-1 / 2}, 0\right)=((0,-x)+A+(0, x)) \tau$. Since $G_{m} / H_{m}$ can be linearly ordered, $\tau$ has an extension $\sigma$ to $G_{m}$ and $H_{m}$ is a $\rho-\sigma$-subgroup of $G_{m}$ [2, Theorem 3.18]. The proof that the extension demonstrated in [2] satisfies $(\delta)$ is similar to the proof of Theorem 3.3 and will be omitted.

If $m<-1$, then a straightforward computation shows that $G_{m}$ is an $R$-group, that is, $n g=n h$ for $n \in N$ implies $g=h$. Since every $R$-group is a power conjugate group (as defined in Theorem 3.1), the class of retractable groups is a proper subclass of the class of power conjugate groups. 
TheOREM 3.8. If $F$ is a free group and $\sigma \in \operatorname{Ret} F$, then every cyclic subgroup of $F$ is a $\sigma$-subgroup.

Proof. By [1, Corollary 2.5], it suffices to show that $\{i, g\} \sigma=g^{n}$ for some $n$ and every $g \in F$. First we assume that if $g=h^{m}$, then $m= \pm 1$. Then the centralizer of $g$ in $F$ is the subgroup generated by $g[8$, p. 42]. By [1, Theorem 2.4] $\{i, g\} \sigma$ belongs to the centralizer of $g$. Hence, the subgroup of $F$ generated by $g$ is a $\sigma$-subgroup. Since every element of $F$ is a power of such a $g$, it follows from the description of Ret $Z$ given prior to Theorem 3.3 that every cyclic subgroup is a $\sigma$-subgroup.

EXAMPLE 3.9. If $G=\left\langle a, c \mid a^{-1} c^{n} a=c^{n}\right\rangle$, where $n$ is a positive even integer, then $G$ is not retractable.

Suppose (by way of contradiction) that $\sigma \in \operatorname{Ret} G$ and let $x=\left\{a, c^{-m} a c^{m}\right\} \sigma$, where $m=n / 2$. Then $c^{-m} x c^{m}=c^{-m}\left(\left\{a, c^{-m} a c^{m}\right\} \sigma\right) c^{m}=$ $\left\{c^{-m} a c^{m}, a\right\} \sigma=2$. Thus, $x$ commutes with $c^{m}$ and it follows that $x=c^{-t}$ for some integer $t$. Then, $i=\left\{a c^{t}, c^{m} a c^{m+t}\right\} \sigma$ and by $[1$, Theorem 2.2], $\left(\alpha c^{t}\right)\left(c^{m} a c^{m+t}\right)=\left(c^{m} a c^{m+t}\right)\left(\alpha c^{t}\right)$. From this relation, we obtain $\left(\alpha c^{m+t} \alpha\right) c^{m}=c^{m}\left(a c^{m+t} a\right)$ and hence, $a c^{m+t} a=c^{s}$, for some integer $s$. In the symmetric group of degree $n+3$, the cycles $c_{1}=(1, \cdots, n)$ and $a_{1}=(n+1, n+2, n+3)$ satisfy the relation $a_{1}^{-1} c_{1}^{n} a_{1}=c_{1}^{n}$ and so the subgroup generated by $a_{1}$ and $c_{1}$ is a homomorphic image of $G$. But then $a_{1} c_{1}^{m+t} a_{1}=c_{1}^{s}$, which is impossible.

We note that Theorems 3.3 and 3.6 have generalizations to splitting extensions of certain torsion free abelian groups by the integers. We close with the following three questions.

(1) Is the group $\left\langle a, c \mid a^{-1} c^{n} a=c^{n}\right\rangle$, where $n$ is an odd positive integer retractable $(n>1)$ ?

(2) Is the group $\left\langle a, c \mid a^{-1} c^{m} a=c^{n}\right\rangle$, where $m, n \in Z \backslash\{-1,0,1\}$ and $\operatorname{gcd}(m, n)=1$, retractable?

(3) If $\sigma$ is a retraction of $G$ that satisfies $(\delta)$, is $G$ an $R$ group?

\section{REFERENCES}

1. R. D. Byrd, J. T. Lloyd, R. A. Mena and J. R. Teller, Retractable groups, Acta Math. Acad. Sci. Hungar. (to appear).

2. - Extensions of group retractions, submitted.

3. - The lattice of solid $\sigma$-subgroups of a retractable group, submitted.

4. P. Conrad, Lattice-ordered groups, Lecture notes, Tulane University, 1970.

5. L. Fuchs and E. Sasiada, Note on orderable groups, Ann. Univ. Sci. Budapest. Eötvös Sect. Math., 7 (1964), 13-17. 
6. M. I. Kargapolov, Completely ordered groups (Russian), Algebra; Logika, 1 (1962), 16-21.

7. A. I. Kokorin, On the theory of completely orderable groups (Russian), Ural. Gos. Univ. Mat. Zap., 4 (1963), 25-29.

8. W. Magnus, A. Karrass and D. Solitar, Combinatorial Group Theory, John Wiley \& Sons, Inc., 1966.

9. D. P. Minassian, Types of fully ordered groups, Amer. Math. Monthly, 80 (1973), 159-169.

Received June 11, 1976 and in revised form April 21, 1977.

UNIVERSITY OF HOUSTON

Houston, TX 77004

AND

UNIVERSITY OF WYOMING

LARAMIE, WY 82071 



\title{
PACIFIC JOURNAL OF MATHEMATICS
}

\section{EDITORS}

\author{
RICHARD ARENS (Managing Editor) \\ University of California \\ Los Angeles, CA 90024 \\ Charles W. Curtis \\ University of Oregon \\ Eugene, OR 97403 \\ C. C. MOORE \\ University of California \\ Berkeley, CA 94720
}

\section{J. DUGUNDJI}

Department of Mathematics

University of Southern California

Los Angeles, CA 90007

R. FINN and J. MILGRAM

Stanford University

Stanford, CA 94305

\section{ASSOCIATE EDITORS}
E. F. BECKENBACH
B. H. NeumanN
F. WOLF
K. YoSHIDA

\section{SUPPORTING INSTITUTIONS}

UNIVERSITY OF BRITISH COLUMBIA

CALIFORNIA INSTITUTE OF TECHNOLOGY

UNIVERSITY OF CALIFORNIA

MONTANA STATE UNIVERSITY

UNIVERSITY OF NEVADA, RENO

NEW MEXICO STATE UNIVERSITY

OREGON STATE UNIVERSITY

UNIVERSITY OF OREGON

OSAKA UNIVERSITY

\author{
UNIVERSITY OF SOUTHERN CALIFORNIA \\ STANFORD UNIVERSITY \\ UNIVERSITY OF HAWAII \\ UNIVERSITY OF TOKYO \\ UNIVERSITY OF UTAH \\ WASHINGTON STATE UNIVERSITY \\ UNIVERSITY OF WASHINGTON \\ $\stackrel{*}{*} \stackrel{*}{*} \stackrel{*}{*}$ AMERICAN MATHEMATICAL SOCIETY
}

The Supporting Institutions listed above contribute to the cost of publication of this Journal, but they are not owners or publishers and have no responsibility for its content or policies.

Mathematical papers intended for publication in the Pacific Jaurnal of Mathematics should be in typed form or offset-reproduced, (not dittoed), double spaced with large margins. Please do not use built up fractions in the text of your manuscript. You may however, use them in the displayed equations. Underline Greek letters in red, German in green, and script in blue. The first paragraph or two must be capable of being used separately as a synopsis of the entire paper. Items of the bibliography should not be cited there unless absolutely necessary, in which case they must be identified by author and Journal, rather than by item number. Manuscripts, in triplicate, may be sent to any one of the editors. Please classify according to the scheme of Math. Reviews, Index to Vol. 39. All other communications should be addressed to the managing editor, or Elaine Barth, University of California, Los Angeles, California, 90024.

The Pacific Journal of Mathematics expects the author's institution to pay page charges, and reserves the right to delay publication for nonpayment of charges in case of financial emergency.

100 reprints are provided free for each article, only if page charges have been substantially paid. Additional copies may be obtained at cost in multiples of 50 .

The Pacific Journal of Mathematics is issued monthly as of January 1966. Regular subscription rate: $\$ 7200$ a year (6 Vols., 12 issues). Special rate: $\$ 36.00$ a year to individual members of supporting institutions.

Subscriptions, orders for back numbers, and changes of address should be sent to Pacific Journal of Mathematics, 103 Highland Boulevard, Berkeley, California, 94708.

PUBLISHED BY PACIFIC JOURNAL OF MATHEMATICS, A NON-PROFIT CORPORATION

Printed at Kokusai Bunken Insatsusha (International Academic Printing Co., Ltd.). 8-8, 3-chome, Takadanobaba, Shinjuku-ku, Tokyo 160, Japan.

Copyright (C) 1975 by Pacific Journal of Mathematics Manufactured and first issued in Japan 


\section{Pacific Journal of Mathematics}

Vol. 72, No. 2

February, 1977

George E. Andrews, Plane partitions. II. The equivalence of the

Bender-Knuth and MacMahon conjectures ................. 283

Lee Wilson Badger, An Ehrenfeucht game for the multivariable quantifiers

of Malitz and some applications ......................... 293

Wayne C. Bell, A decomposition of additive set functions ............ 305

Bruce Blackadar, Infinite tensor products of $C^{*}$-algebras ............. 313

Arne Brøndsted, The inner aperture of a convex set .............. 335

N. Burgoyne, Finite groups with Chevalley-type components........... 341

Richard Dowell Byrd, Justin Thomas Lloyd and Roberto A. Mena, On the retractability of some one-relator groups .....................

Paul Robert Chernoff, Schrödinger and Dirac operators with singular potentials and hyperbolic equations .................... 361

John J. F. Fournier, Sharpness in Young's inequality for convolution ....... 383

Stanley Phillip Franklin and Barbara V. Smith Thomas, On the metrizability

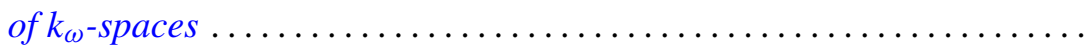

David Andrew Gay, Andrew McDaniel and William Yslas Vélez, Partially normal radical extensions of the rationals .................... 403

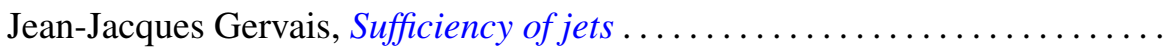

Kenneth R. Goodearl, Completions of regular rings. II . .

Sarah J. Gottlieb, Algebraic automorphisms of algebraic groups with stable maximal tori

Donald Gordon James, Invariant submodules of unimodular Hermitian forms.....

J. Kyle, $W_{\delta}(T)$ is convex.

Ernest A. Michael and Mary Ellen Rudin, A note on Eberlein compacts ...

Ernest A. Michael and Mary Ellen Rudin, Another note on Eberlein compacts ....

Thomas Bourque Muenzenberger and Raymond Earl Smithson, Fixed point theorems for acyclic and dendritic spaces.

Budh Singh Nashier and A. R. Rajwade, Determination of a unique solution of the quadratic partition for primes $p \equiv 1(\bmod 7)$.

Frederick J. Scott, New partial asymptotic stability results for nonlinear ordinary differential equations ....................

Frank Servedio, Affine open orbits, reductive isotropy groups, and dominant gradient morphisms; a theorem of Mikio Sato..........

D. Suryanarayana, On the distribution of some generalized square-full integers.................................. 